\title{
Calculation of phase diagrams of binary fec and ideal hep alloys undergoing ordering transitions
}

\author{
ANANYA GHOSH MOULIC, G V S SASTRY and S LELE \\ Centre of Advanced Study in Metallurgy, Department of Metallurgical Engineering, Banaras \\ Hindu University, Varanasi $22100 j$, India \\ MS received 5 February 1994; revised 2 May 1994

\begin{abstract}
The tetrahedron approximation of the cluster variation method (CVM) has been employed to investigate phase diagrams having fcc-based ordered and disordered phases. This approximation is also applicable to the binary hcp ordered structures with ideal axial ratio. The CVM developed by Kikuchi consists of calculating approximate expressions for the number of configurations and hence entropy of a crystal lattice having definite distribution of clusters (points, pairs, triangles, tetrahedra, etc.) of lattice points which in general may be occupied by one of a given set of atomic species. Tetrahedral multi-atom interactions denoted by $\alpha$ and $\beta$ are utilized for expressing the configurational energy. The equilibrium cluster distribution is then found by minimizing the free energy by utilizing the natural iteration method developed by Kikuchi. The effect of $\alpha$ and $\beta$ parameters on the topology of the resulting phase diagrams is observed by assigning several negative and positive values to them. The invariant reactions were also determined in each case. Finally a study was made on the $\mathrm{Cd}-\mathrm{Mg}$ diagram.
\end{abstract}

Keywords. Cluster; sublattice; cluster variation method; configurational energy; configurational entropy; phase diagrams; invariant reactions.

\section{Introduction}

The tendency for ordering in alloys is opposed by thermal agitation. For each temperature, there is a characteristic equilibrium degree of order at which the opposing tendencies are just balanced. The aim of any theory of ordering is the development of a relation which helps in constructing the order-disorder boundaries in phase diagrams. For this, we require an appropriate physical model of ordering. Earlier models based on point (Bragg and Williams 1934) and pair correlations (Bethe 1935) were insufficient for predicting a topologically correct phase diagram. The cluster variation method (CVM) (Kikuchi 1951) for calculating entropy has shown that the point and pair correlations are part of a hierarchy of approximations (i.e. clusters made up of points, pairs, triangles, etc.). Local order can be treated adequately by considering three-dimensional clusters. With the incorporation of multi-atom interactions in the configurational energy (van Baal 1973), CVM has become even more versatile in performing approximate but increasingly accurate calculations.

CVM in the tetrahedral approximation has been used to model the $\mathrm{Cu}-\mathrm{Au}$ (fcc) phase diagram (Kikuchi and de Fontaine 1978). The calculated diagram was in qualitative agreement with the observed one. A similar study has been undertaken for Fe-Al (bcc) (Inden and Pepperhoff 1990). Pitsch and Inden (1991) have reviewed the subject recently.

In the present work CVM in the tetrahedron approximation with multi-atom interactions has been applied to calculate phase diagrams for binary systems exhibiting $\mathrm{L}_{2}$ and $\mathrm{L} 1_{0}$ ordered structures apart from the disordered fcc structure. This 
approximation is also applicable to the binary hcp $\left(\mathrm{DO}_{19}, \mathrm{~B} 19\right)$ case when the axial ratio is ideal. The entire range of multi-atom interaction parameters $(\alpha, \beta)$ corresponding to the stability of $\mathrm{Ll}_{2}, \mathrm{Ll}_{0}$ and disordered phases has been selected for study. The effect of varying $\alpha$ and $\beta$ on the topology of the phase diagrams as well as the invariant reactions has been investigated. Finally, optimum $\alpha$ and $\beta$ values have been found for the Cd-Mg phase diagram.

\section{Theoretical formulation}

CVM (Kikuchi 1951) coupled with the natural iteration method (NIM) (Kikuchi 1974) has been applied to calculate the internal energy as well as the configurational entropy for binary alloys with fcc structures. These calculations are also applicable to hcp structures with ideal axial ratio.

\subsection{Cluster variation method}

The first step in CVM is the selection of a basic figure called a motif (Kikuchi 1951). A motif is a three-dimensional arrangement of atoms in a crystal structure. Earlier workers (Bragg and Williams 1934; Bethe 1935) have shown that choice of smaller motifs led to loss of accuracy while computational labour increases almost exponentially for larger motifs. Hence, keeping a fair balance between accuracy and ease of computation, the basic three-dimensional motif chosen was a regular tetrahedron. This motif is also valid for hcp structures with ideal axial ratio.

Various types of clusters and subclusters are obtained by populating the sites of the motif by A or B atoms. For a point motif, there are two types of point clusters with the motif sites $(a, b, c, d)$ being occupied by $\mathbf{A}$ or $\mathbf{B}$ atoms respectively: $x_{\mathrm{A}}^{i}$, the probability of occupancy of the $i$ site by $A$ atom; $x_{B}^{i}$ the probability of occupancy of the $i$ site by B atoms.

Hence

$$
x_{\mathrm{A}}^{i}+x_{\mathrm{B}}^{i}=1
$$

The next higher set of clusters is formed by pair motif. The pair cluster variables are of four types: (i) $y_{\mathrm{AA}}^{i j}$, the probability of occurrence of AA bonds with A atoms on $i$ and $j$ sites; (ii) $y_{\mathrm{BA}}^{i j}$, the probability of occurrence of BA bonds with $\mathrm{B}$ atom on $i$ site and $\mathrm{A}$ atom on $j$ site; (iii) $y_{\mathrm{AB}}^{i j}$, the probability of occurrence of $\mathrm{AB}$ bonds with $\mathrm{A}$ atom on $i$ site and $\mathrm{B}$ atom on $j$ site; and (iv) $y_{\mathrm{BB}}^{i j}$, the probability of occurrence of BB bonds with B atoms on both $i$ and $j$ sites. Hence

$$
y_{\mathrm{AA}}^{i j}+y_{\mathrm{BA}}^{i j}+y_{\mathrm{AB}}^{i j}+y_{\mathrm{BB}}^{i j}=1 \text {. }
$$

The point variables can be expressed in terms of these pair variables by relations of the type (Kikuchi 1951)

$$
x_{\mathrm{A}}^{i}=y_{\mathrm{AA}}^{i j}+y_{\mathrm{AB}}^{i j} .
$$

Next in succession is the triangular cluster. We do not assign any variable to this as in the tetrahedron approximation there is no contribution to the entropy from the triangular clusters (Kikuchi 1951). 
The next higher cluster is based on a tetrahedral motif. On populating the four lattice sites by $A$ and B atoms we get 16 possible tetrahedral cluster variables. Symmetry considerations reduce this number to 5 with appropriate 'weights' attached to each of them. These weights are equal to the number of identical configurations in different orientations of the same cluster. The tetrahedral clusters are denoted by $Z_{m n p q}^{a b c d} . Z_{m n p q}^{a b c d}$ is defined as a distribution variable for the configuration $m-n-p-q$ of the tetrahedron, where $m, n, p, q$ correspond to an A or B atom; $a, b, c, d$ are the corresponding lattice sites. As an example $Z_{B A A A}^{a b c d}$ is the probability of occurrence of tetrahedra with one $B$ atom at the $a$ site. The normalization relation is

$$
\sum_{m, n, p, q} Z_{m n p q}^{a b c d}=1
$$

The pair and point variables can be expressed in terms of the tetrahedral cluster variables (Kikuchi 1951). As an example,

$$
\begin{aligned}
y_{\mathrm{AA}}^{a b}=Z_{\mathrm{AAAA}}^{a b c d}+Z_{\mathrm{AABA}}^{a b c d}+Z_{\mathrm{AAAB}}^{a b c d}+Z_{\mathrm{AABB}}^{a b c d}, & \\
x_{\mathrm{A}}^{a}=Z_{\mathrm{AAAA}}^{a b c d}+Z_{\mathrm{AABA}}^{a b c d}+Z_{\mathrm{ABAA}}^{a b c d}+Z_{\mathrm{AAAB}}^{a b c d}+Z_{\mathrm{AABB}}^{a b c d}+Z_{\mathrm{ABBA}}^{a b c d} & +Z_{\mathrm{ABAB}}^{a b c d}+Z_{\mathrm{ABBB}}^{a b c d}
\end{aligned}
$$

Due to preferential occupation of atoms on certain sites during ordering, it is necessary to define sublattices to describe the system completely. In the ordered fcc/hcp (ideal axial ratio) structure, the unit cell can be described in terms of four interpenetrating simple cubic/hcp sublattices. Figure 2 shows the basal plane projection of hcp structure showing four sublattices $a, b, c, d$. In figure 1 the tetrahedral motif is identified within the hcp cell.

Depending on the state of order in the structure, two or more of these sublattices may be identically occupied by $A$ or $B$ atoms. In the $A_{3} B$ (and $A B_{3}$ ) type of ordering

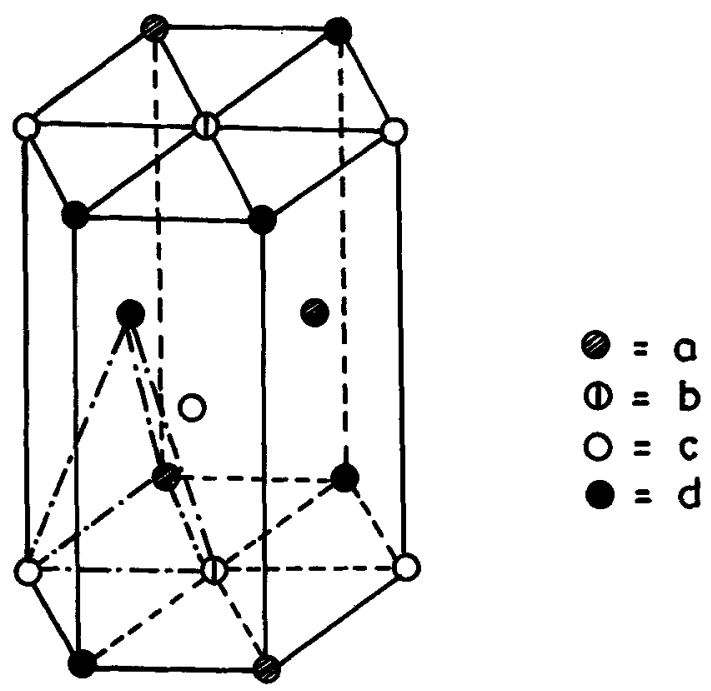

Figure 1. Three-dimensional view of the hcp structure with the tetrahedral motif shown by -..- lines. 


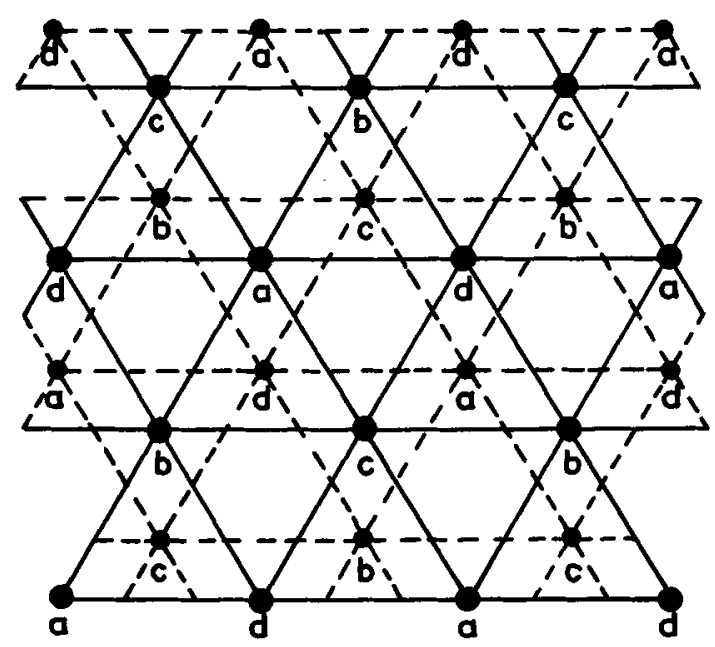

Figure 2. Basal plane projection of hcp structure showing four sublattices $(a, b, c, d)$ per layer. Continuous lines connect sites on the plane of the paper and broken lines connect the ones above and below the plane of the paper.

( $\mathrm{LL}_{2}$ and $\mathrm{DO}_{19}$ ), atomic sites $a, b, c$ are equivalent while $d$ site is distinct. In the $\mathrm{AB}$ type of ordering ( $\mathrm{Ll}_{0}$ and $\left.\mathrm{B} 19\right)$, the sites $a$ and $b$ are equivalent and so are $c$ and $d$. In the disordered case, the sublattice distinction is entirely lost, i.e. $a \equiv b \equiv c \equiv d$ although there can still be some short-range order (SRO).

CVM consists in calculating the approximate expressions for the number of configurations, $\omega$, of a structure having definite distribution of clusters (points, pairs, triangles, tetrahedra, etc. as described above) of atomic sites which in general may be occupied by one of a given set of atomic species. The equilibrium cluster distribution is then found by minimizing the Helmholtz free energy, $F$ :

$$
\begin{aligned}
& F=U-T S, \\
& F=U-k T \ln \omega,
\end{aligned}
$$

where $U$ is the configurational energy, $S$ the configurational entropy, $T$ the temperature, and $k$ the Boltzmann constant.

2.1a Configurational energy: The configurational energy $U$ can be expressed as a linear function of the cluster fractions (Kikuchi 1951). It is assumed that each tetrahedron, pair or atom in the structure has a certain amount of energy. This energy is assumed to depend on the kind, number and position of atoms that make up the cluster, and not on the sublattice sites on which the atoms happen to be situated. This term is given by (van Baal 1973)

$$
U=2 N\left[4 Z_{\mathrm{AAAB}}^{a b c d}\left\{\frac{3}{2}(1+\alpha) W\right\}+6 Z_{\mathrm{AABB}}^{a b c d}(2 W)+4 Z_{\mathrm{ABBB}}^{a b c d}\left\{\frac{3}{2}(1+\beta) W\right\}\right]
$$

where $N$ is the number of lattice sites, $W$ the effective pair interaction parameter, and $\alpha$ and $\beta$ are multi atom interaction parameters. 
2.1b Configurational entropy: The configurational entropy $S$ of the alloys was first calculated by Kikuchi (1951). According to the Kikuchi approximation the configurational entropy $S$ can be written in terms of the tetrahedral entropy $S_{z}$, corrected for overlaps by pair entropy $S_{y}$ and point entropy $S_{x}$ terms

$$
S=S_{z}-S_{y}-5 S_{x}
$$

More explicitly the equation can be written as

$$
\begin{aligned}
S=- & 2 N k\left[\sum_{m, n, p, q} Z_{m n p q}^{a b c d} \ln Z_{m n p q}^{a b c d}\right]+N k\left[\sum_{i j} \sum_{m, n} \ln y_{m n}^{i j}\right] \\
& -\frac{5}{4} N k\left[\sum_{i} \sum_{m} x_{m}^{i} \ln x_{m}^{i}\right] .
\end{aligned}
$$

The free energy $F$ in (7) can now be found by substituting the configurational energy $U$ and configurational entropy $S$. The equilibrium cluster distribution is obtained by minimizing the free energy with respect to the tetrahedral cluster variables.

However, a more convenient way of deriving the equilibrium state is by the minimization of the grand potential, $G$ with respect to the $Z s$, keeping the chemical potentials, $\mu_{\mathrm{A}}\left(=-\mu_{\mathrm{B}}\right)$, fixed (Kikuchi et al 1977).

The conditions for the coexistence of the phases is obtained by determining the compositions, for which the equality of chemical potentials hold. Hence for calculating phase diagrams we minimize the grand potential $\Omega$ :

$$
\Omega=U-T S-\sum_{m} N_{m} \mu_{m}=U-T S-N \sum_{m} x_{m} \mu_{m}
$$

where $\mu_{m}$ is the chemical potential of atomic species $m$ and $x_{m}$ the fraction of atomic species.

Hence

$$
\begin{aligned}
\mathbf{\Omega}= & U+N k T\left[\sum_{m, n, p, q} Z_{m n p q}^{a b c d} \ln Z_{m n p q}^{a b c d}-\sum_{i j} \sum_{m, n} y_{m n}^{i j} \ln y_{m n}^{i j}\right. \\
& \left.+\frac{5}{4} \sum_{i} \sum_{m} x_{m}^{i} \ln x_{m}^{i}\right]+N \lambda\left[1-\sum_{m, n, p, q} Z_{m n p q}^{a b c d}\right] \\
& -\frac{N}{4}\left[\mu_{\mathrm{A}}\left\{\sum_{i} x_{\mathrm{A}}^{i}\right\}+\mu_{\mathrm{B}}\left\{\sum_{i} x_{\mathrm{B}}^{i}\right\}\right],
\end{aligned}
$$

where $\lambda$ is the Lagrangian multiplier. Minimizing $\Omega$ with respect to the $Z$ s we get

$$
Z_{m n p q}^{i j k l}=\exp \left[\frac{1}{k T}\left(\frac{\lambda}{2}-\varepsilon_{m n p q}-\frac{\left(\mu_{m}+\mu_{n}+\mu_{p}+\mu_{q}\right.}{8}\right)\right] y^{1 / 2} \cdot X^{-5 / 8},
$$

where $y=$ product of pair variables $=y_{m n}^{(i j)} \cdot y_{m p}^{(i k)} \cdot y_{m q}^{(i l)} \cdot y_{n p}^{(j k)} \cdot y_{n q}^{(j l)} y_{p q}^{(k l)}, X=$ product of point variables $=x_{m}^{(i)} \cdot x_{n}^{(j)} \cdot x_{p}^{(k)} \cdot x_{q}^{(l)}$, and $\varepsilon_{m n p q}=$ tetrahedral cluster energies.

Solving the above type of nonlinear equations gives the equilibrium state of order. 
In the disordered case there are five such equilibrium equations. In the $A_{3} B$ (and $A B_{3}$ ) case, there are eight equilibrium equations. In the $A B$ case there are nine equilibrium equations.

\subsection{Natural iteration method}

The nonlinear simultaneous equations resulting from CVM formulation are solved by NIM (Kikuchi 1974). The several steps of the method are described below:

(i) The values of $\mu_{\mathrm{A}}$ and $1 / k T$ are selected and the first input values of the $x \mathrm{~s}$ are guessed.

(ii) The $y$ s of the initial guess are found from $y_{m n}=x_{m} \cdot x_{n}$, which is based on the assumption of random distribution.

(iii) The $x s$ and $y$ s are used to calculate initial $Z_{m n p q}^{a b c d}$ and thus the normalization factor $\exp [\lambda / 2 k T]$ from (4).

(iv) The normalization factor is then used to calculate the output $Z_{m n p q}^{\text {abcd }}$ s.

(v) The next inputs of $x$ s and $y s$ are calculated from the geometric reduction relations

(5) and (6) for the next iteration cycle.

This iterative process using the above steps is the NIM. After the iterations have converged, the Lagrangian multiplier, $\lambda$, appearing in the normalization factor becomes equal to the grand potential per lattice point $(\lambda=G / N)$.

\section{Results}

\subsection{Effect of multi-atom interaction parameters on topology of phase diagram}

Following Cahn and Kikuchi (1979), for $W<0$, the phases $A_{3} B, A B$ and $A_{3}$ are stable within a triangular domain $X Y Z$ (figure 3) of parameter space with vertices at $\mathrm{X}: \alpha=-\frac{1}{3}, \beta=-\frac{1}{3} ; \mathrm{Y}: \alpha=-\frac{1}{3}, \beta=1 ;$ and $\mathrm{Z}: \alpha=1, \beta=-1 / 3$. At the points $\mathrm{X}, \mathrm{Y}$ and $Z$ respectively only the structures $A B, A B_{3}$ and $A_{3} B$ are stable as single phases. Selection of $\alpha, \beta$ values along the lines $\mathrm{XY}, \mathrm{YZ}$ and $\mathrm{XZ}$ would give rise to phase diagrams in which only two phases out of $A B, A B_{3}$ and $A_{3} B$ would be present and the phases $A, B, A B$ and $A B_{3}$, respectively would be missing. We wish to construct theoretical phase diagrams in which three phase fields corresponding to $A B, A B_{3}$ and $A_{3} B$ phases are present in addition to that for the disordered phase. Evidently the $\alpha, \beta$ values should be chosen in such a way that they lie within the triangle XYZ. A starting point of selection naturally would be $(0,0)$, i.e. point $O$. The line joining $\mathrm{O}$ to $\mathrm{X}$ when extended to the opposite side divides the triangle into two symmetric regions. Thus it is sufficient to explore $\alpha, \beta$ values to one side of the line PQ. Above the line $\mathrm{PQ}$, the $\alpha, \beta$ values give rise to phase diagrams which are topologically similar to those obtained below the line $\mathrm{PQ}$ except that the roles of $\mathrm{A}$ and $\mathrm{B}$ components are interchanged. Then the triangle $P Q R$ was constructed by choosing points symmetric to $O$ which are also well within the triangle $X Y Z$. Seven such points associated with the triangle give rise to seven pairs of parametric values $(\alpha, \beta)$.

Theoretical phase diagrams were calculated using these values of the parameters and the invariant reactions were determined in each case. Equal values of $\alpha$ and $\beta$ resulted in symmetric phase diagrams. The results are shown in table 1 . Three types 
of invariant reactions have been observed and are given below. Of the three, two are of eutectoid type:

Eutectoid I: Disordered $\rightarrow A B+A_{3} B$ or $A_{3}$,

Eutectoid II: $\mathbf{A}_{\mathbf{3}} \mathrm{B} \rightarrow$ Disordered $+\mathrm{AB}$,

and the third is a peritectoid reaction

Peritectoid: Disordered $+\mathrm{AB} \rightarrow \mathrm{A}_{3} \mathrm{~B}$ or $\mathrm{AB}_{3}$.

Several inferences have been drawn. These are depicted pictorially for easy visualization in figure 4.

Table 1. Nature of invariant reactions for various values of $\alpha$ and $\beta$.

\begin{tabular}{|c|c|c|c|c|c|}
\hline \multirow{2}{*}{$\begin{array}{l}\text { Point in } \\
\text { parameter } \\
\text { space }\end{array}$} & \multirow[b]{2}{*}{$\alpha$} & \multirow[b]{2}{*}{$\beta$} & \multicolumn{2}{|c|}{ Nature of invariant reactions } & \multirow{2}{*}{$\begin{array}{l}\text { Corresponding } \\
\text { phase diagram }\end{array}$} \\
\hline & & & $X_{B}<0.5$ & $X_{\mathrm{B}}>0.5$ & \\
\hline 0 & 0 & 0 & Eutectoid I & Eutectoid I & (figure 5) \\
\hline $\mathbf{P}$ & $-\frac{1}{6}$ & $-\frac{1}{6}$ & Peritectoid & Peritectoid & (figure 6) \\
\hline $\mathbf{Q}$ & $\frac{\mathbf{b}}{6}$ & & Eutectoid I & Eutectoid I & (figure 7) \\
\hline $\mathbf{R}$ & $\frac{1}{2}$ & $-\frac{i}{6}$ & Eutectoid II & Peritectoid & (figure 9) \\
\hline $\mathbf{s}$ & $\frac{2}{6}$ & $-\frac{6}{6}$ & Eutectoid I & Peritectoid & (figure 12) \\
\hline $\mathbf{T}$ & $\frac{1}{3}$ & 0 & Eutectoid II & Eutectoid I & (figure 8) \\
\hline C & $\begin{array}{l}\frac{3}{6} \\
\frac{1}{6}\end{array}$ & $-\frac{1}{18}$ & Eutectoid I & Peritectoid & (figure 11) \\
\hline
\end{tabular}

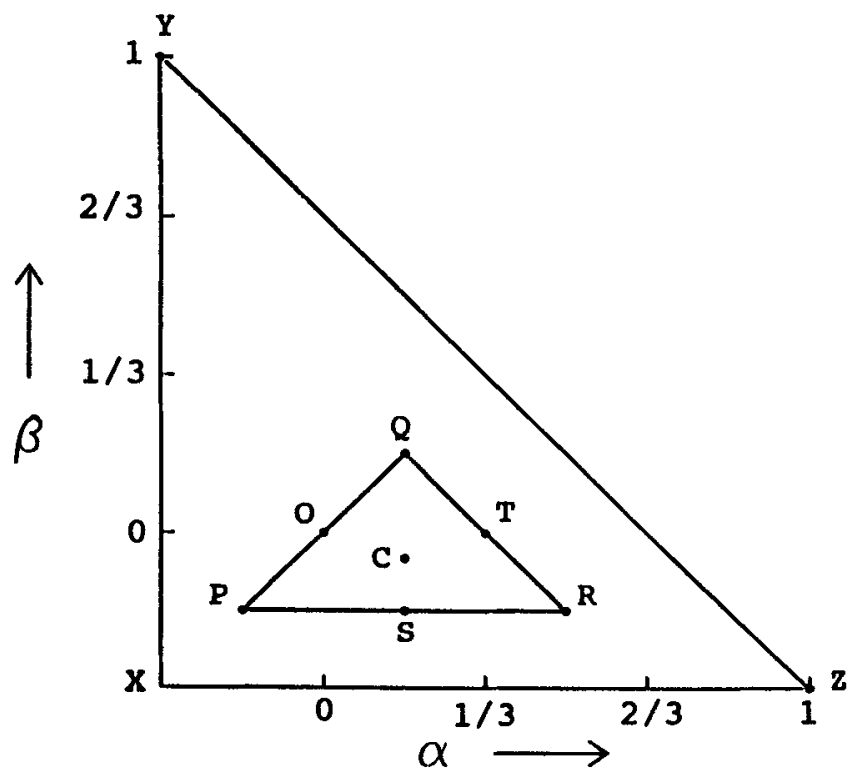

Figure 3. Stability region of $\mathrm{L}_{2} / \mathrm{DO}_{19}$ and $\mathrm{L1}_{0} / \mathrm{B} 19$ phases in the $\alpha, \beta$ parameter space. $\mathrm{O}, \mathrm{P}, \mathrm{Q}, \mathrm{R}, \mathrm{S}, \mathrm{T}$ and $\mathrm{C}$ correspond to the $\alpha, \beta$ values utilized for calculating phase diagrams. 

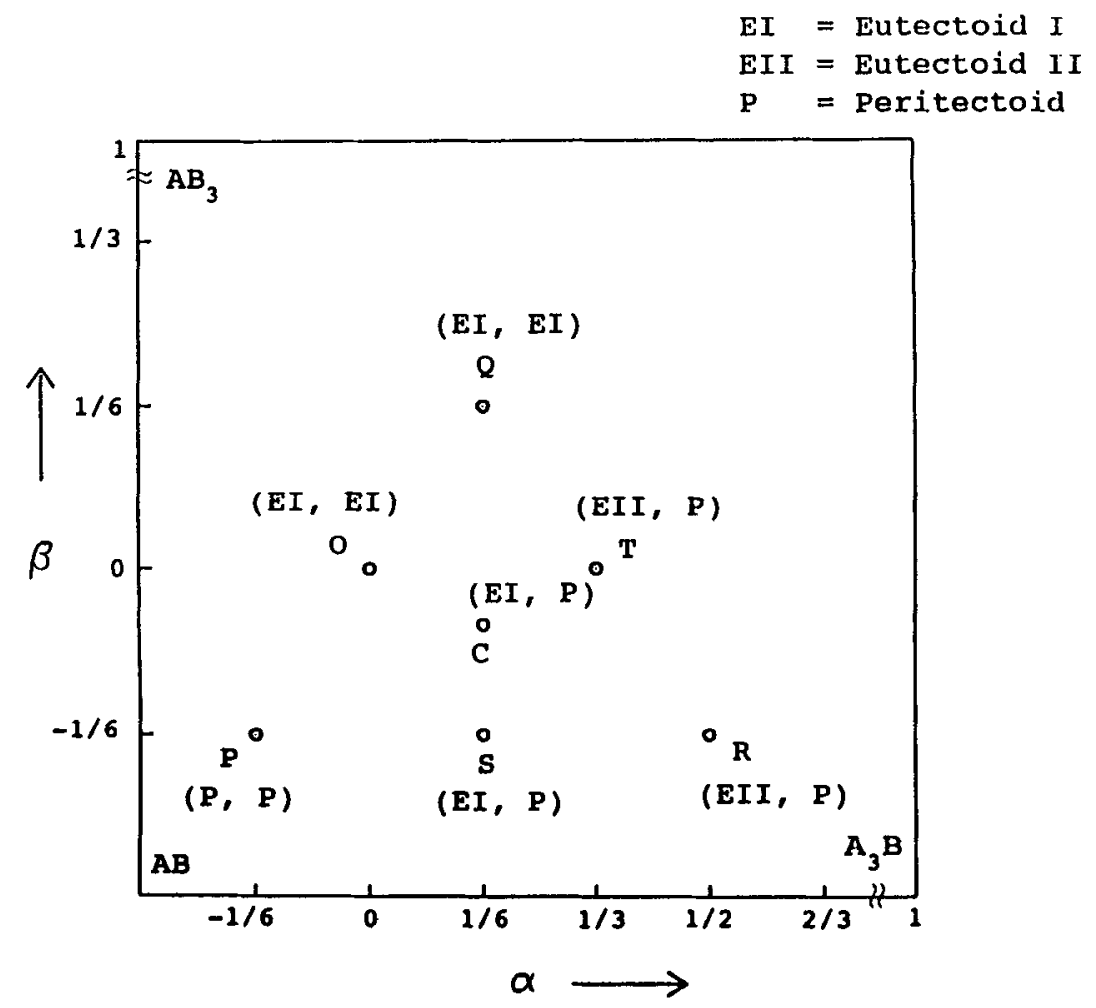

Figure 4. Representation of invariant reactions for different $\alpha, \beta$ parameters.

The invariant reactions mentioned earlier are denoted by EI, EII and P respectively. Phases stable at three of the corners of the parameter space are also shown.

According to figure 4 , it is seen that

(i) In moving from $O(\alpha=0, \beta=0$ in figure 5), to $P(P$ : $\alpha=-1 / 6, \beta=-1 / 6)$, the eutectoid invariant (EI) at $O$ changes to peritectoid invariant at $P$. The $A B$ phase field is stabilized relative to $A_{3} B$ and $A_{3}$ (figure 6 ).

(ii) Along $\mathrm{OQ}(\mathrm{Q}: \alpha=1 / 6, \beta=1 / 6)$, the eutectoid invariant (EI) remains unchanged. The $A_{3} B$ and $A B_{3}$ phase fields are stabilized relative to $A B$ from $O$ to $Q$ (figure 7). (iii) Along $\mathrm{OT}(\mathrm{T}: \alpha=1 / 3, \beta=0)$, one of the type I eutectoid invariants changes to eutectoid type II (for compositions below 50\% B) while the other changes to a peritectoid (for compositions above $50 \% \mathrm{~B}$ ). At $\mathrm{T}, \mathrm{A}_{3} \mathrm{~B}$ becomes the most stable phase and $\mathrm{AB}$ the least stable one (figure 8 ).

(iv) Along $\mathrm{OR}\left(\mathrm{R}: \alpha=\frac{1}{2}, \beta=-\frac{1}{6}\right.$ ) the changes are qualitatively similar to those along OT. Here again $A_{3} B$ is the most stable of the three phases, but both $A B$ and $A B_{3}$ phases are present in very small amounts (figure 9). As an example, the details of the eutectoid II-type invariant reaction (for compositions below $50 \%$ B) has been shown in figure 10. The peculiarity of this reaction is that an ordered phase transforms to a disordered and another ordered phase.

(v) Along OC (C: $\alpha=1 / 6, \beta=-1 / 18)$, the eutectoid I transformation occurs below $50 \% \mathrm{~B}$ and peritectoid transformation above $50 \% \mathrm{~B}$. In this case $\mathrm{A}_{3} \mathrm{~B}$ phase is 


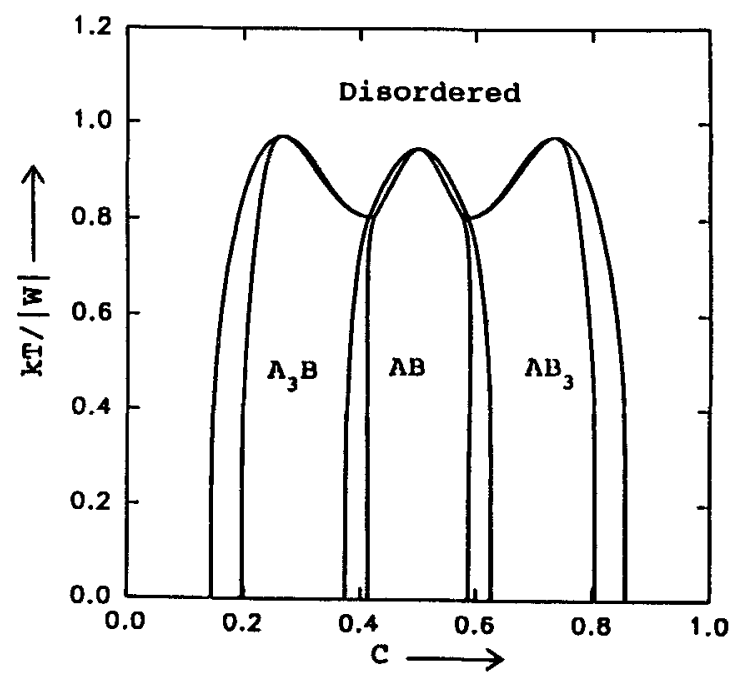

Figure 5. Phase diagram for $\alpha=\beta=0$.

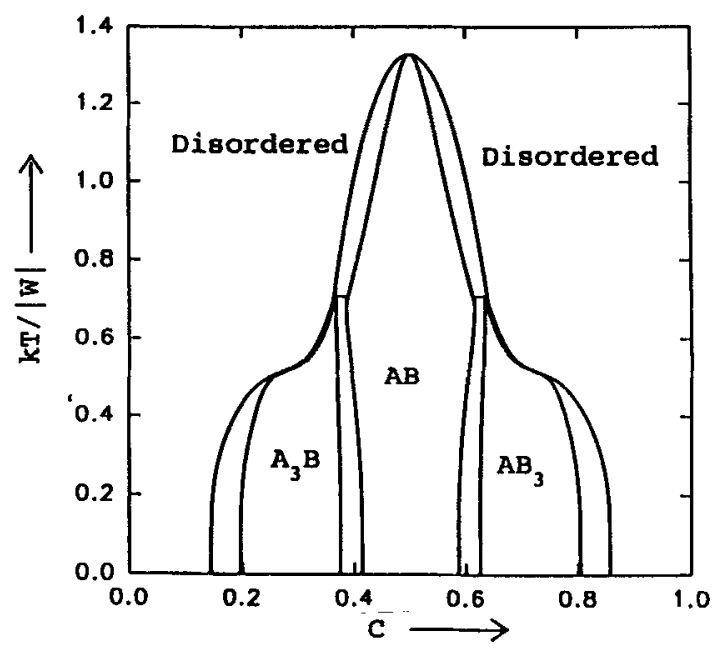

Figure 6. Phase diagram for $\alpha=\beta=-1 / 6$.

stabilized. $A B$ and $A_{3}$ phase fields are present in nearly equal amounts (figure 11). (vi) Along $\mathrm{OS}(\mathrm{S}: \alpha=1 / 6, \beta=-1 / 6)$ the invariants are similar to those along $\mathrm{OC}$. The stability of the phases decreases as we go from $A_{3} B$ to $A B$ and finally $A B_{3}$ (figure 12).

\subsection{Calculation of the $\mathrm{Cd}-\mathrm{Mg}$ phase diagram}

Part of the experimentally observed $\mathrm{Cd}-\mathrm{Mg}$ phase diagram is reproduced in figure 13a (Moser et al 1984). Comparison with figures 5 to 12 shows that none of the calculated phase diagrams matches the observed one too well. However, we can approximately match the peritectoid temperatures $\left(T_{1} \approx 125^{\circ} \mathrm{C}\right.$ and $\left.T_{2} \approx 186^{\circ} \mathrm{C}\right)$ and the transition temperature $\left(T_{t} \approx 253^{\circ} \mathrm{C}\right)$. Since both invariants are peritectoids, the parameter values 


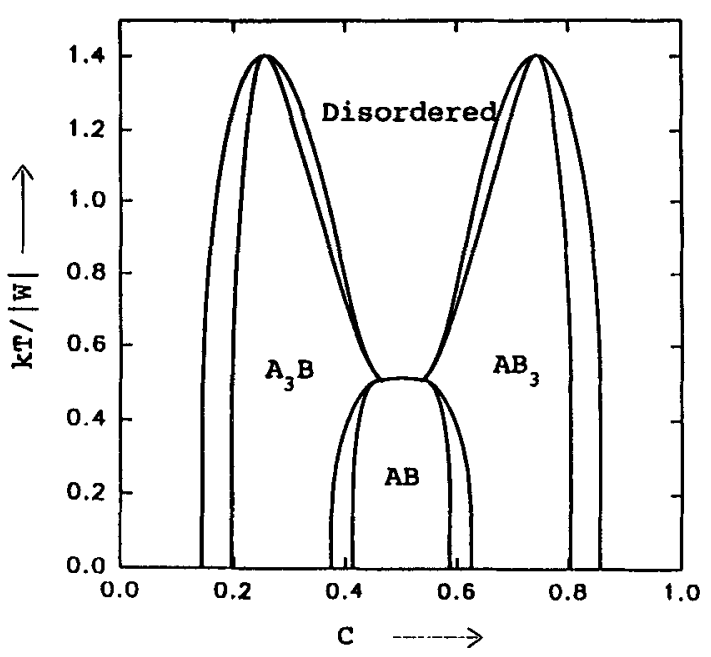

Figure 7. Phase diagram for $\alpha=\beta=1 / 6$.

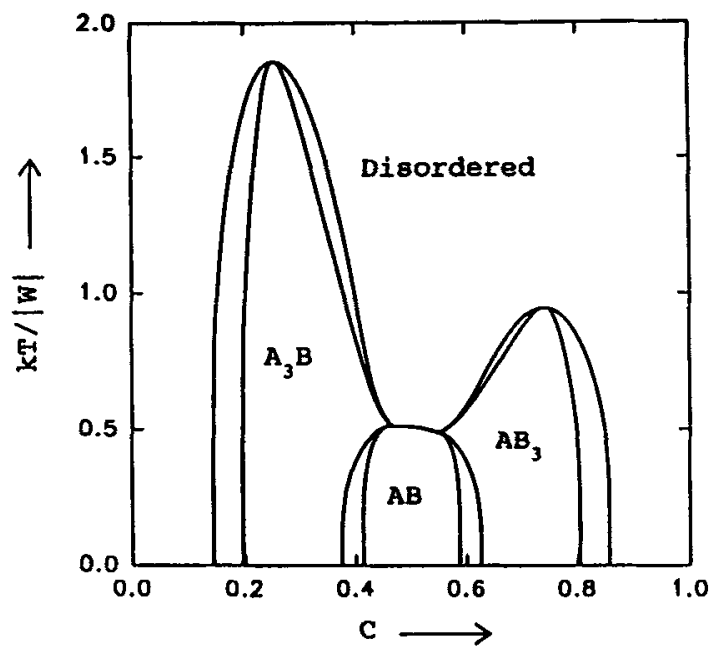

Figure 8. Phase diagram for $\alpha=1 / 3, \beta=0$.

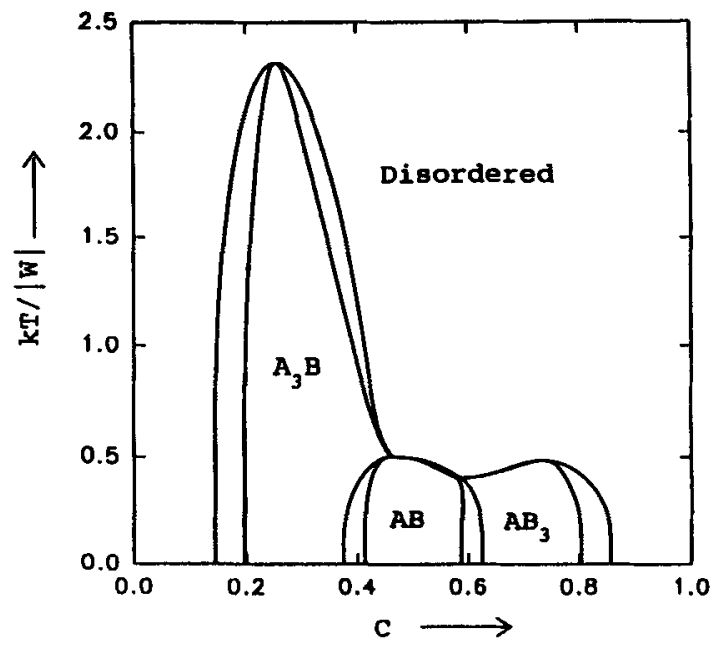

Figure 9. Phase diagram for $\alpha=1 / 2, \beta=-1 / 6$. 


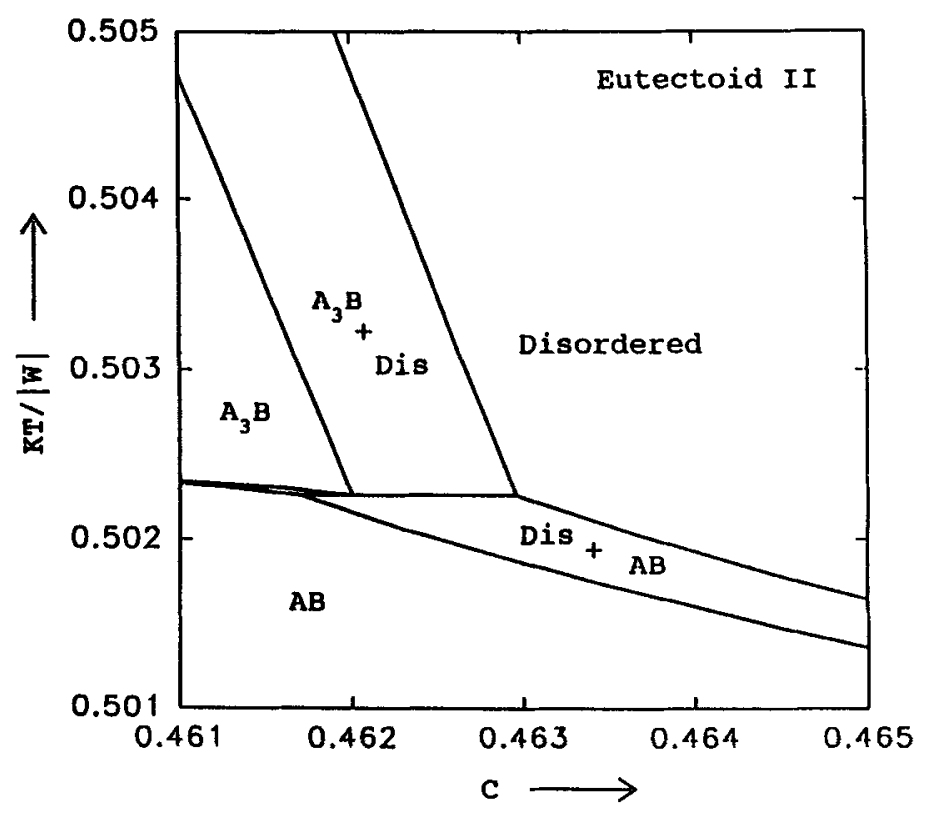

Figure 10. Eutectoid II-type invariant reaction (below $50 \%$ B) for $\alpha=1 / 2, \beta=-1 / 6$.

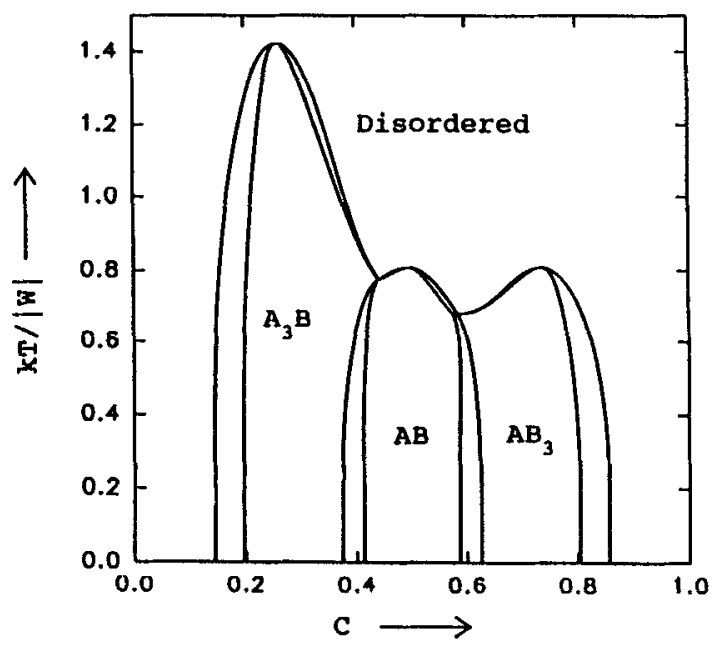

Figure 11. Phase diagram for $\alpha=1 / 6, \beta=-1 / 18$.

should be close to the line OP (figure 4). By trial and error optimum match could be obtained for $\alpha=\angle 0.07, \beta=-0.01$ (figure 13b). It was observed that from $\alpha=\beta=-0.08$ to $\alpha=\beta=-0.01$, the theoretically obtained phase diagrams showed a maximum as well as a minimum in the $A_{3} B$ region. This type of behaviour is absent in the $\mathrm{Cd}-\mathrm{Mg}$ phase diagram. A further refinement of the $\alpha$ and $\beta$ to larger number of significant digits is possible but has not been attempted in view of the difference in features exhibited by the experimental and theoretical phase diagrams.

The agreement between the calculated and the observed phase diagrams can be 


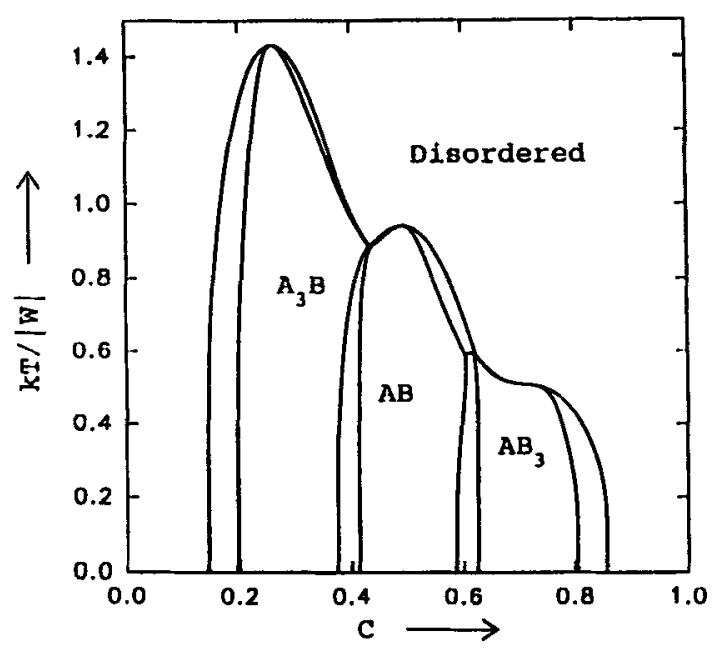

Figure 12. Phase diagram for $\alpha=1 / 6, \beta=-1 / 6$.
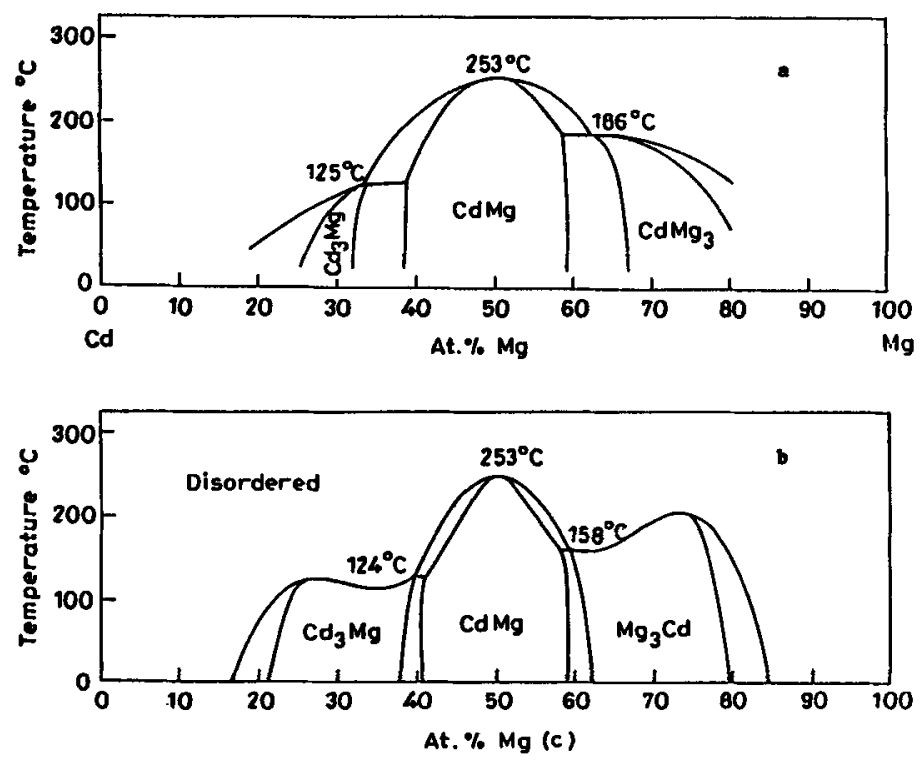

Figure 13. (a) Experimentally observed $\mathrm{Cd}-\mathrm{Mg}$ phase diagram. (b) Theoretical phase boundaries of $\mathrm{Cd}-\mathrm{Mg}$ calculated for $\alpha=-0.07, \beta=-0.01$.

improved by a consideration of (i) non ideal axial ratio, (ii) a larger motif, say the tetrahedron octahedron ( $\mathrm{T}-\mathrm{O}$ ) motif which has been found to give results agreeing very well with Monte Carlo results (Mohri et al 1985), and (iii) taking account of higher-neighbour interactions (Mohri et al 1985; Asta et al 1993).

\section{Conclusion}

CVM in the tetrahedral approximation has been utilized to calculate phase diagrams for a range of multiatom interaction parameter values. Asymmetry in the phase 
diagrams is achieved by making the multiatom interaction parameters ( $\alpha$ and $\beta$ ) unequal. These multiatom forces give rise to a variety of topologically distinct phase diagrams. The calculated and the observed phase diagrams for $\mathrm{Cd}-\mathrm{Mg}$ show the same qualitative features. However, the quantitative agreement is unsatisfactory.

\section{Acknowledgements}

The authors wish to acknowledge financial support from the Council of Scientific and Industrial Research, New Delhi, through a grant-in-aid scheme. They also acknowledge helpful discussions with Dr A K Singh, Shri V Lele, Shri B N Sarma and Shri P Ghosal.

\section{References}

Asta M, McCormack R and de Fontaine D 1993 Phys. Rev. B48 748

Bethe H 1935 Proc. R. Soc. (London) A150 552

Bragg W L and Williams E J 1934 Proc. R. Soc. (London) A145 669

Cahn J W and Kikuchi R 1979 Acta Metall. 271329

Cenedese P and Cahn J W 1994 Prog. Theor. Phys. Suppl. 11595

Inden $\mathrm{G}$ and Pepperhoff W $1990 \mathrm{Z}$. Metallkde 80770

Kikuchi R 1951 Phys. Rev. 81988

Kikuchi R 1974 J. Chem. Phys. 601071

Kikuchi R, de Fontaine D, Murakami M and Nakamura T 1977 Acta Metall. 25207

Kikuchi R and de Fontaine D 1978 NBS Special Publication 496967

Mohri T, Sanchez J M and de Fontaine D 1985 Acta Metall. 331171

Moser Z, Gasior W, Wypartowicz J and Zabdyr L 1984 in Binary alloy phase diagrams (ed. in chief) T B Massalski (Metals Park:ASM) Vol. 1, p 666

Pitsch W and Inden G 1991 in Phase transformation in materials (ed.) P Haasen, Vol. 5, Materials science and technology, (eds.) R W Cahn, P Haasen and E J Kramer (Weinheim : VCH) p 497

van Baal C M 1973 Physica 64571

\section{Note added in proof}

It has been shown by Cenedese and Cahn (1994), that ideal hcp and fcc structures having identical interaction energies have free energy differences which are very small but significant. The calculations reported in this work are thus strictly applicable only to fcc structures. However, results for ideal hcp structures are expected to show only small differences. 